\title{
Compact Microring-Based Wavelength-Selective Inline Optical Reflector
}

\author{
George T. Paloczi, Student Member, IEEE, Jacob Scheuer, Member, IEEE, and Amnon Yariv, Life Fellow, IEEE
}

\begin{abstract}
We present a novel design for a compact planar integrated optic reflector based on a microring resonator add-drop wavelength-selective filter. Good agreement is found between the theoretical expectation and the measurements of a device fabricated in optical polymer. The measured device exhibits better than 10-dB rejection for wavelengths resonant with the microring.
\end{abstract}

Index Terms-Dielectric resonator filters, integrated optics, microresonators, optical polymers.

\section{INTRODUCTION}

$\mathbf{O}$ RIGINALLY proposed in 1969 for channel dropping in optical communications systems [1], microring and microdisk resonators have recently become the subject of intense research for a variety of applications [2]-[11], [14]-[17]. The original ideas of wavelength routing and switching have been extended, resulting in several synthesis and analysis techniques for high-order filtering using multiple resonators [2], [3]. Microrings have been used to demonstrate optical modulation using the electrooptic effect in polymer [4], and for all-optical switching using free-carrier injection in $\mathrm{GaAs}-\mathrm{AlGaAs}$ [5]. In virtue of their long photon lifetimes, high quality-factor resonators may be applied as optical delay lines [6] and for low threshold lasers [7]. More recently, interest in biological sensing has led to the development of photonic biosensors based on microring resonators [8].

Here we present a novel geometry for wavelength-selective reflectors employing microring resonators, in the spirit of [9] and [10]. Inline reflectors, particularly fiber Bragg gratings [11], are widely used components in optical telecommunications systems. Applications include external wavelength stabilization for lasers, gain-flattening, dispersion-compensation, and add-drop filtering. Integration of inline reflectors into planar optical circuits is important for several reasons. Integrated reflectors can be much smaller than corresponding fiber devices and can be fabricated in a variety of materials with special nonlinear functionality or with specific dispersion properties. In addition, integrated optical reflectors can be combined on a single chip with additional photonic devices such as modulators, couplers, sources, etc. However, integration of Bragg gratings within a planar waveguide optical circuit is difficult due to the highresolution lithography required over large areas necessary to

Manuscript received August 10, 2004; revised October 1, 2004. This work was supported in part by the Defense Advanced Research Project Agency and in part by the National Science Foundation.

The authors are with the Department of Applied Physics, California Institute of Technology, Pasadena, CA 91125 USA (e-mail: paloczi@caltech.edu; koby@caltech.edu; ayariv@caltech.edu).

Digital Object Identifier 10.1109/LPT.2004.839448

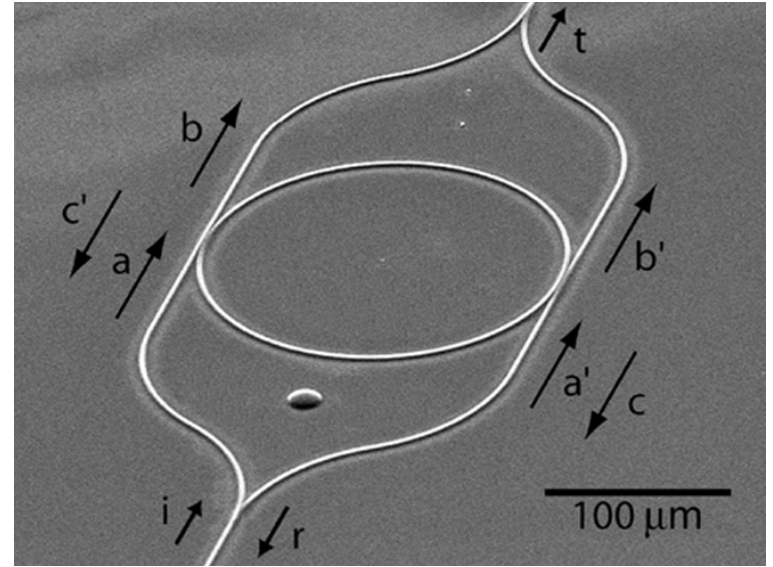

Fig. 1. Scanning electron microscope perspective image of a device similar to that measured here. The input, reflected, and transmitted signals are labeled $i$, $r$, and $t$, respectively. Two sets of fields, $a, b, c$ and the corresponding primes obey (2) and (3).

achieve high reflection efficiency and low scattering loss [12]. This is especially the case in integrated geometries requiring aperiodic gratings. An alternative to Bragg gratings for planar integrated optic inline reflectors is shown in Fig. 1. In this letter, we present the design, fabrication, and characterization of a microring-based inline integrated optical reflector. We discuss the potential applications and various advantages of the device.

\section{THEORY}

Referring to the device shown in Fig. 1, the central portion between the $Y$ junctions is the well-known microring resonator add-drop filter. Each of the two waveguides interacts with the ring according to the coupling matrix [13]

$$
T=\left[\begin{array}{cc}
t & \kappa \\
-k^{*} & t^{*}
\end{array}\right]
$$

where, for a unity input, $t$ is the field amplitude transmitted past the coupler, and $\kappa$ is the field amplitude coupled across the coupler. For a lossless coupler, $|t|^{2}+|\kappa|^{2}=1$. In general, the two couplers need not be the same, but in the following we assume identical couplers.

Given an input $a$, as shown in Fig. 1, the normalized intensity past the resonator is given by [14]

$$
\left|\frac{b}{a}\right|^{2}=\frac{|t|^{2}+|t|^{2} \alpha^{2}-2|t|^{2} \alpha \cos (\theta)}{1+|t|^{4} \alpha^{2}-2|t|^{2} \alpha \cos (\theta)}
$$

where $\alpha$ is the field amplitude remaining after one round-trip in the resonator, and $\theta=\beta \cdot L$ is the phase accumulated over a ring 


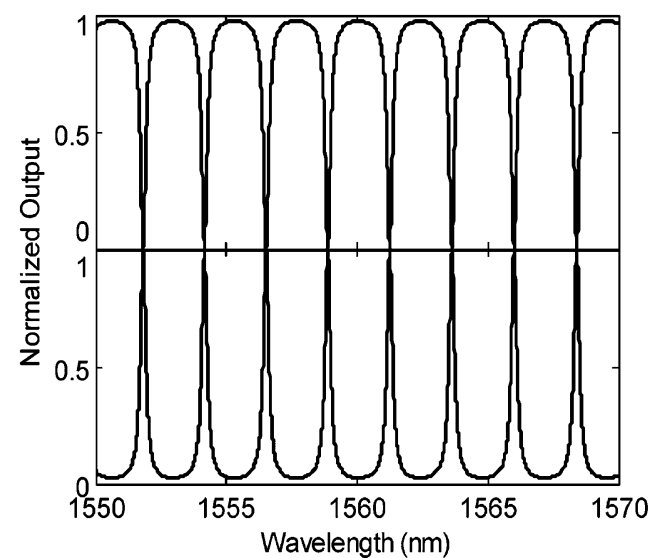

Fig. 2. Normalized transmission (upper) and reflection (lower) signals for the microring-based inline reflector. For the calculation, the microring radius is $108.25 \mu \mathrm{m}$, the effective refractive index is $1.515, t$ is 0.85 , and the loss parameter $\alpha$ is taken to be 1 .

circumference $L$ for a waveguide with propagation constant $\beta$. The normalized intensity of the field exiting the "drop" port of the add-drop filter is given by [14]

$$
\left|\frac{c}{a}\right|^{2}=\frac{\left(1-|t|^{2}\right)^{2} \alpha^{2}}{1+|t|^{4} \alpha^{2}-2|t|^{2} \alpha \cos (\theta)} .
$$

When $\theta=\beta \cdot L$ is an integer multiple of $2 \pi$, and if the loss term $\alpha$ is close to one, then the input light exits through the "drop" port and no light is transmitted past the resonator.

As shown in Fig. 1, a $Y$ junction splitter equally divides the input light between the upper and lower arms of the device. Thus, there are two identical sets of fields $\left(a, b, c\right.$, and $a^{\prime}, b^{\prime}$, $c^{\prime}$ ) obeying (2) and (3). Since the "drop" ports are connected to the input, the dropped signals of (3) are the reflected signals. Fig. 2 shows the predicted transmitted signal (upper plot) and the reflected signal (lower plot) for parameters as described in the figure caption.

\section{EXPERIMENT}

For demonstration purposes, the microring-based inline reflector was fabricated in optical polymer. A silicon wafer with 5 - $\mu \mathrm{m}$ thermal oxide $(n=1.445)$ served as the device substrate and lower cladding layer. The core polymer SU-8 $(n=1.565$, available from Microchem Corp.) was cross-linked using direct electron-beam exposure. The waveguides were $2 \mu \mathrm{m}$ in width and $1.8 \mu \mathrm{m}$ in thickness. Finite-difference mode solver calculations predict a well-confined first-order mode $\left(n_{\text {eff }}=1.515\right)$ and weakly guided second- and third-order modes around $1550 \mathrm{~nm}$. The microring resonator radius was $108 \mu \mathrm{m}$. The separation between the waveguides and the microring were designed to be $250 \mathrm{~nm}$, resulting in approximately $25 \%$ power coupling. For measurement, the end-facets were prepared by cleaving the substrate wafer.

Transverse electric polarized light from a tunable laser was fiber-coupled to the device after passing through a circulator. Index matching fluid was applied, covering both the device and the fiber input, to reduce the reflected signal noise to an acceptably low level. The reflected signal was collected by connecting

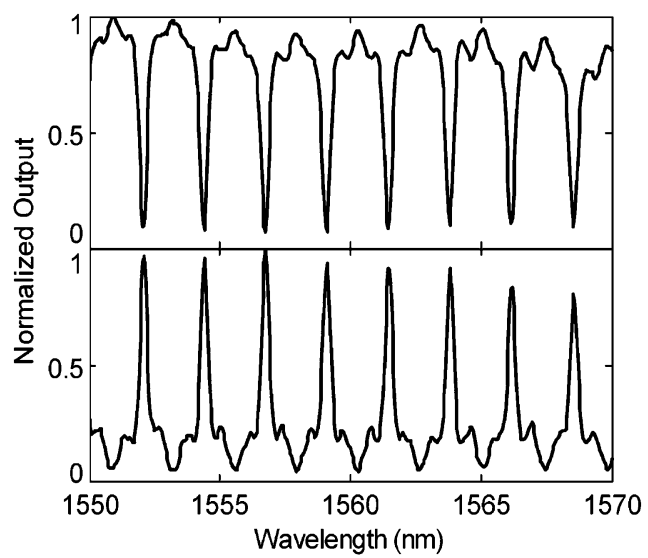

Fig. 3. Measurement results showing the normalized transmission (upper) and reflection (lower) signals. The extinction ratios for the nulls in the transmission signal are better than $10 \mathrm{~dB}$.

the reflection port of the circulator to a photodetector. The transmitted signal was collected by focusing the output on a photodetector using a microscope objective. The wavelength was scanned from 1550 to $1570 \mathrm{~nm}$ and the normalized transmitted (upper plot) and reflected (lower plot) signals were recorded, as shown in Fig. 3.

Comparing the theoretical fit in Fig. 2 to the measurement in Fig. 3, we find good agreement. As expected, the nulls of the transmitted signal occur at the same wavelengths as the reflected peaks. The rejection ratio of the transmitted signal nulls is better than $10 \mathrm{~dB}$. These nulls appear every free spectral range (FSR) of $2.33 \mathrm{~nm}$. A closer inspection of the smaller features in the data shows a high degree of complementarity between the transmitted and reflected signals. These smaller features were caused by the weakly guided higher order modes. This is supported by similar multimode behavior previously observed in slightly thicker SU-8 waveguides, and the lack of such features in slightly thinner waveguides [15]. A best-fit of the data suggests a propagation loss of approximately $15 \mathrm{~dB} / \mathrm{cm}$, however, in the demonstration there was no attempt to minimize the propagation loss. Since the bend radii are large, we attribute the propagation loss predominantly to scattering loss. This is reduced by stabilization of the lithography or by postfabrication annealing of the polymer.

\section{DISCUSSION AND CONCLUSION}

As an alternative to an integrated Bragg grating reflection filter, the microring-based reflector presents several advantages and increased flexibility. Most notably, the microring-based reflector is more compact than typical integrated waveguide gratings, allowing for simpler and more controlled fabrication, as well as for the possibility of incorporation with additional photonic devices.

The ability to simply manipulate the lineshape of the transmitted and reflected responses presents a significant advantage over both Bragg grating structures and the ring-based reflector suggested in [9]. As previously pointed out, there exist several synthesis techniques for constructing higher order filter responses using multiple microrings [2], [3]. Applying these algorithms to the reflectors presented here, one can design a system 


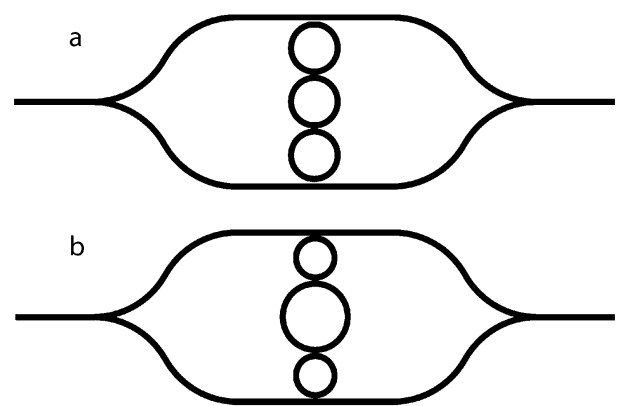

Fig. 4. (a) Reflector employing three microring resonators to exhibit a special spectral response, for instance, a wavelength-flattened reflection band. (b) Reflector employing the Vernier effect, with resonators of different radii.

to deliver the desired lineshape transmission and reflection. One restriction is that there must always be an odd number of resonators for the device to act as a reflector. Shown schematically in Fig. 4(a) is such a device, employing three microrings to achieve a third-order response.

Along similar lines, composite structures involving different resonators can be used, as illustrated in Fig. 4(b). When resonators of different circumference are placed in series, only wavelengths that are resonant with all resonators are transmitted in steady state. Thus, the reflected peaks and the transmitted nulls will only occur at wavelengths for which $m \cdot \mathrm{FSR}_{1}=$ $n \cdot \mathrm{FSR}_{2}=\ldots$ (the Vernier effect) for integers $m, n, \ldots$ [16]. By employing this effect, integrated inline reflectors with large FSRs can be devised.

Finally, these reflective devices are easily rendered tunable by using the thermooptic or electrooptic effects. These can be exploited to either tune the lineshape in multiple resonator structures, or to alter the resonant wavelength of an individual reflector. These tunable reflective elements can serve as laser end mirrors, providing wavelength selectivity as in [17].

In conclusion, simple wavelength-selective inline reflectors based on microring resonator add-drop filters have been presented. The concept was demonstrated in optical polymer. The resulting device exhibited a rejection ratio better than $10 \mathrm{~dB}$. The measured transmission and reflection signals were observed to have a high degree of complementarity, as expected from theoretical considerations. The versatility of these inline reflectors lies in the fact that multiple rings can be used for higher order responses or to employ the Vernier effect. The compact struc- tures can be fabricated using conventional techniques and, thus, are readily incorporated with other photonic devices. Use of thermooptic or electrooptic effects renders the reflected wavelengths or lineshapes tunable for use as laser end-mirrors.

\section{REFERENCES}

[1] E. A. J. Marcatili, "Bends in dielectric optical waveguides," Bell Syst. Technol. J., vol. 48, no. 7, pp. 2103-2103, 1969.

[2] R. Orta, P. Savi, R. Tascone, and D. Trinchero, "Synthesis of multiplering-resonator filters for optical systems," IEEE Photon. Technol. Lett., vol. 7, no. 12, pp. 1447-1449, Dec. 1995.

[3] B. E. Little, S. T. Chu, H. A. Haus, J. Foresi, and J.-P. Laine, "Microring resonator channel dropping filters," J. Lightw. Technol., vol. 15, no. 6, pp. 998-1005, Jun. 1997.

[4] P. Rabiei, W. H. Steier, C. Zhang, and L. R. Dalton, "Polymer microring filters and modulators," J. Lightw. Technol., vol. 20, no. 11, pp. 1968-1975, Nov. 2002.

[5] T. A. Ibrahim, W. Cao, Y. Kim, J. La, J. Goldhar, P.-T. Ho, and C. H. Lee, "All-optical switching in a laterally coupled microring resonator by carrier injection," IEEE Photon. Technol. Lett., vol. 15, no. 1, pp. 36-38, Jan. 2003

[6] G. Lenz, B. J. Eggleton, C. K. Madsen, and R. E. Slusher, "Optical delay lines based on optical filters," IEEE J. Quantum Electron., vol. 37, no. 4, pp. 525-532, Apr. 2001.

[7] S. L. McCall, A. F. J. Levi, R. E. Slusher, S. J. Pearton, and R. A. Logan, "Whispering gallery mode microdisk lasers," Appl. Phys. Lett., vol. 60, no. 3, pp. 289-291, Jan. 1992.

[8] R. W. Boyd and J. E. Heebner, "Sensitive disk resonator photonic biosensor," Appl. Opt., vol. 40, no. 31, pp. 5742-5747, Nov. 2001.

[9] B. E. Little, S. T. Chu, and H. A. Haus, "Second-order filtering and sensing with partially coupled traveling waves in a single resonator," Opt. Lett., vol. 23, no. 20, pp. 1570-1572, Oct. 1998.

[10] J. K. S. Poon, J. Scheuer, and A. Yariv, "Wavelength-selective reflector based on a circular array of coupled microring resonators," IEEE Photon. Technol. Lett., vol. 16, no. 5, pp. 1331-1333, May 2004.

[11] A. Othonos, "Fiber Bragg gratings," Rev. Sci. Instrum., vol. 68, no. 12, pp. 4309-4341, Dec. 1997.

[12] M. H. Lim, T. E. Murphy, J. Ferrera, J. N. Damask, and H. I. Smith, "Fabrication techniques for grating-based optical devices," J. Vac. Sci. Technol. B, vol. 17, no. 6, pp. 3208-3211, Nov./Dec. 1999.

[13] A. Yariv, "Universal relations for coupling of optical power between microresonators and dielectric waveguides," Electron. Lett., vol. 36, no. 4, pp. 321-322, Feb. 2000.

[14] J. M. Choi, R. K. Lee, and A. Yariv, "Ring fiber resonators based on fused-fiber grating add-drop filters: Application to resonator coupling," Opt. Lett., vol. 27, no. 18, pp. 1598-1600, Sep. 2002.

[15] G. T. Paloczi, "Fabrication and measurement of SU-8 waveguides," . unpublished.

[16] Y. Yanagase, S. Suzuki, Y. Kokubun, and S. T. Chu, "Box-like filter response and expansion of FSR by vertically triple coupled microring resonator filter," J. Lightw. Technol., vol. 20, no. 8, pp. 1525-1529, Aug. 2002.

[17] B. Liu, A. Shakouri, and J. E. Bowers, "Passive microring-resonatorcoupled lasers," Appl. Phys. Lett., vol. 79, no. 22, pp. 3561-3563, Nov. 2001. 